\title{
Structural Cell Biology: Preparing Specimens for Cryo-Electron Tomography Using Focused-Ion-Beam Milling
}

\author{
Elizabeth Villa ${ }^{1,2}$, Miroslava Schaffer ${ }^{2}$, Ben Engel ${ }^{2}$, Jürgen Plitzko ${ }^{2,3}$ and Wolfgang Baumeister ${ }^{2}$ \\ ${ }^{1}$ Department of Chemistry and Biochemistry, University of California San Diego, La Jolla, USA. \\ 2 Department of Molecular Structural Biology, Max Planck Institute of Biochemistry, Martinsried, \\ Germany. \\ ${ }^{3}$ Bijvoet Center for Biomolecular Research, Utrecht University, Utrecht, The Netherlands.
}

Cryo-electron microscopy has established itself as a central tool in structural biology, ideally suited to study large macromolecular complexes from in vitro samples. Cryo-electron tomography (CET) can extend the applicability of cryo-electron microscopy to study these complexes in situ. Recent technological advances allow CET to be applied to questions in cell biology that go beyond structural determination, where a quantitative analysis of cellular features is required including molecular distribution and supramolecular architecture. However, the ability of CET to provide three-dimensional landscapes of cells is limited by the thickness of the sample that can be examined in situ with a resolution that is high enough for the identification of molecular species. For intermediate resolution electron microscopes, samples must be of less than $500 \mathrm{~nm}$ in thickness. Most cells, specially eukaryotes, exceed the thickness accessible to CET, limiting its routine application to isolated or reconstituted subcellular systems, small prokaryotic cells, or thin peripheral regions of cells.

We have developed a workflow that uses focused-ion-beam milling at cryo temperatures (cryo-FIB) to produce samples of adequate thickness for CET from frozen-hydrated biological cells [1,3]. This novel approach is applicable to all types of cells grown or deposited directly on EM grids [2] by generating $100-400 \mathrm{~nm}$ lamellae that are supported by the surrounding cellular material, and thus remain in the EM grid. The grid is then transferred out of the dual beam and stored at liquid nitrogen temperatures for subsequent imaging in a cryo-TEM. The routine use of this methodology will be described in detail, along with its application to the study of the structure of macromolecular complexes that were previously inaccessible to structural studies in their native environment inside the cell.

As an example application, the structural dynamics of the nuclear pore complex (NPC) will be presented. The NPC is one of the largest macromolecular machines in the cell, composed of hundreds of proteins, selectively controlling all traffic between the nucleus and the cytoplasm. Its architecture is central to understanding nuclear transport. However, due to its sheer size, its local environment and its dynamic nature, determining its structure at molecular resolution remains a challenge for conventional techniques. The determination of the NPC architecture using cryo-FIB has led to the first structural study of the NPC in its native environment, free of the distortions caused by purification.

\section{References:}

[1] A Rigort et al, PNAS 109 (2012), p. 444.

[2] E Villa et al, Current Opinion in Structural Biology, 23 (2014), p. 77.

[3] M Marko et al, Nat Methods 4(3) (2007) p.215.

[4] Work was supported by the European Commission's 7th Framework Programme grant agreement HEALTH-F4-2008-201648/PROSPECTS, the Deutsche Forschungsgemeinschaft Excellence Cluster 
CIPSM and SFB 1035 (to WB) and GRK 1721 (to EV), the Federal Ministry of Education and Research $(\mathrm{BMBF})$, and from an inter-institutional research initiative of the Max Planck Society.
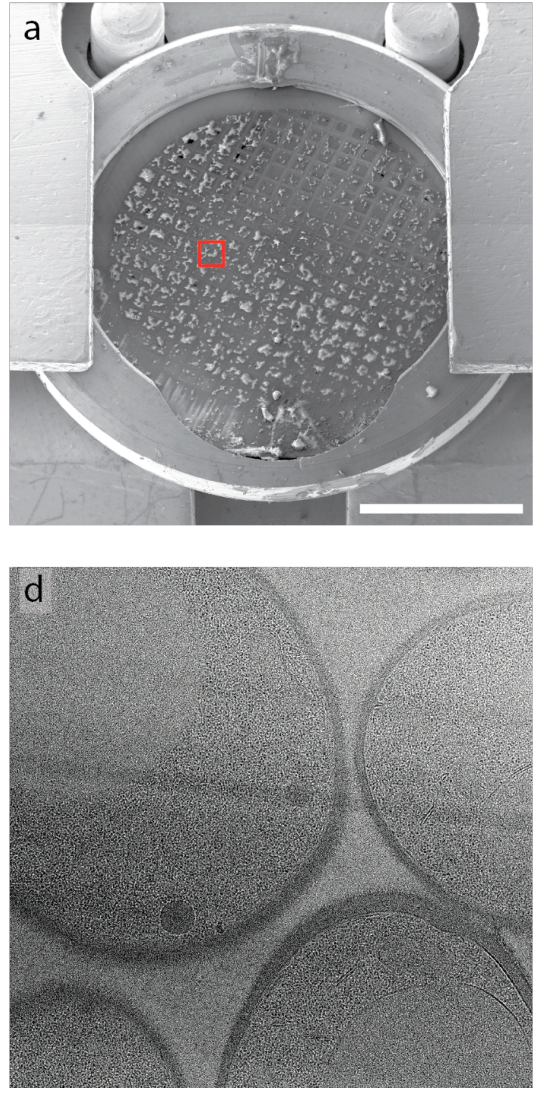
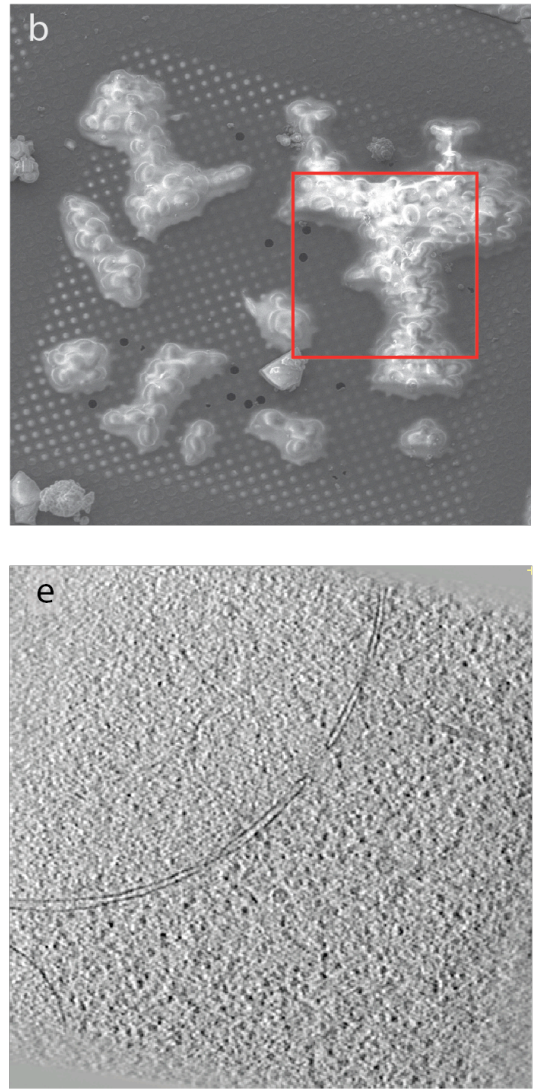
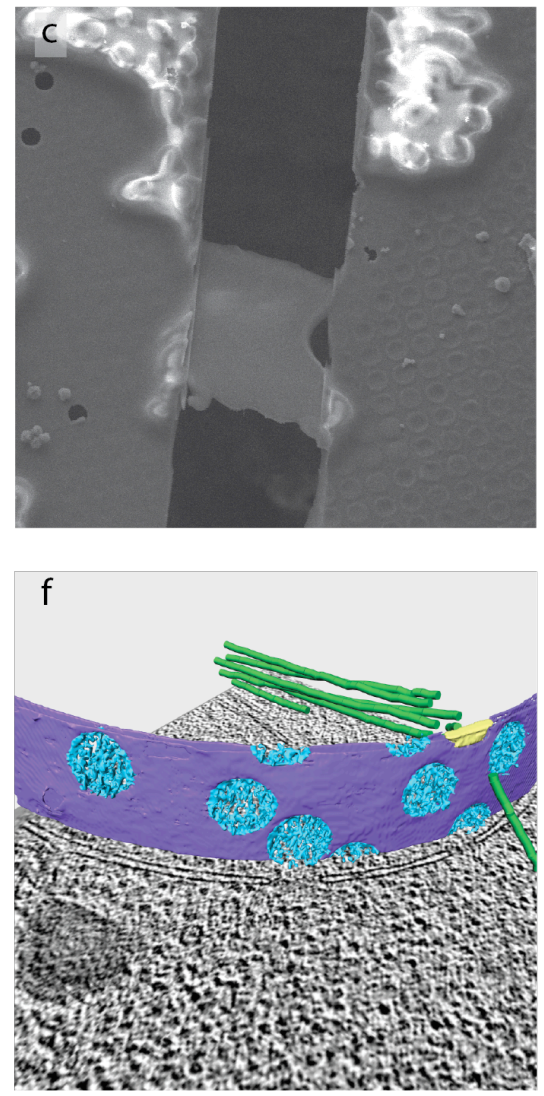

Figure 1. (a) TEM grid mounted in a modified autogrid cryo-holder with frozen-hydrated yeast cells. (b) SEM image of yeast cells on a TEM grid. (c) SEM image of a lamella after cryo-FIB milling the region highlighted in b. (d) Cryo-transmission electron micrograph of a section of a yeast cell prepared with cryo-FIB. (e) Cryo-electron tomogram of a yeast cell. (f) Segmented densities of the nuclear envelope (purple), nuclear pore complex (cyan) and microtubules (green) of the tomogram in (e). 